\title{
NGHIÊN CÚU PHƯƠNG PHÁP PHÂN LOẠI HƯỚNG ĐỐI TƯợNG TRÊN TỦ LIẸU ẢNH MÁY BAY KHÔNG NGƯỜI LÁI
}

\author{
LÊ VŨ HŌNG HẢI(1), ĐÕ TH! HOÀl(2), VŨ KỲ LONG(3) \\ ${ }^{(1)}$ Học viện Kỹ thuật Quân sự, (2) Viện Khoa học Đo đạc và Bản đồ \\ (3) Sở Tài nguyên và Môi trường Hà Nội
}

\section{Tóm tắt:}

Hệ thống máy bay không người lái (UAVs - Unmanned Aerial Vehicle system) thu thập thông tin ở tầm thấp nên tư liệu ảnh UAV cho phép quan sát rõ đặc tính của vật thể và phục vụ giải đoán bằng mắt. Tư liệu viễn thám từ hệ thống UAV thiếu các thông tin về phổ nhưng lại nhiều thông tin về hình dạng và cấu trúc của đối tượng. Tuy nhiên hiện còn ít các nghiên cứu phân tích thông tin bề mặt mặt đất từ ảnh hàng không hay ảnh UAV do phương pháp phân loại dựa trên giá trị pixel cho kết quả thấp. Trong nội dung bài báo, tác giả trình bày kết quả nghiên cứu phương pháp phân loại hướng đối tượng bằng phần mềm eCognition 9.0 trên tư liệu ảnh UAV với độ phân giải $40 \mathrm{~cm}$. Phương pháp phân loại hướng đối tượng cho phép tách được các đối tượng trên ảnh độ phân giải siêu cao dựa trên những đặc điểm hình dạng đặc trưng với độ chính xác cao.

\section{1. Đặt vấn đề}

Phân loại ảnh là một quá trình cơ bản của xử lý ảnh số. Thuật ngữ thông thường phân loại ảnh thường được hiểu là quá trình phân chia ảnh thành những phân lớp khác nhau. Hiện nay, việc chiết tách thông tin từ dữ liệu viễn thám chủ yếu sử dụng hai phương pháp chính: (1) Phương pháp phân loại dựa vào đặc trưng phổ của từng điểm ảnh (pixel-based) thường được sử dụng để phân loại ảnh có độ phân giải thấp và trung bình; (2) Phương pháp phân loại hướng đối tượng (object-based) được phát triển và sử dụng để phân loại ảnh có độ phân giải cao và siêu cao như vệ tinh SPOT độ phân giải $2.5 \mathrm{~m}$, đến độ phân giải siêu cao (Very-high resolution) như vệ tinh QuickBird độ phân giải $0.6 \mathrm{~m}$, WorldView-2 độ phân giải $0.46 \mathrm{~m}$, WorldView-4 độ phân giải $0.31 \mathrm{~m} .$. đặc biệt là ảnh chụp từ ảnh máy bay không người lái có độ phân giải đến $\mathrm{cm}$ (tham khảo Hình 1).

Phương pháp phân loại hướng đối tượng dựa trên tập hợp các điểm ảnh có giá trị đồng nhất về phổ, các thông tin hình học và mối quan hệ giữa các đối tượng [1], [5], [3]. Tư liệu ảnh vệ tinh độ phân giải cao hoặc siêu cao thường có nhiều kênh phổ và chứa nhiều thông tin về đặc trưng phản xạ phổ của các đối tượng lớp phủ. Tuy nhiên, tư liệu ảnh chụp từ máy bay hoặc thiết bị bay không người lái thường có ít thông tin về phổ (chỉ gồm 3 kênh đỏ, xanh lục, xanh chàm) nhưng có nhiều thông tin về hình dạng, cấu trúc của đối tượng do chụp ở độ cao thấp. Do đó, phương pháp phân loại hướng đối tượng đang được các nhà khoa học nghiên cứu thử nghiệm trên tư liệu ảnh UAV với độ phân giải tới $\mathrm{cm}$ và cho độ chính xác cao [6], [3], [8].

Các nhà khoa học trong nước cũng đã có nghiên cứu về phương pháp phân loại hướng đối tượng. Trịnh Thị Hoài Thu thử nghiệm trên ảnh WorldView-2 với độ chính xác đạt $84.4 \%$ [6]; Phạm Văn Duẩn nghiên cứu chỉ số khoanh vi phù hợp trong phân loại hướng đối tượng trên ảnh vệ tinh SPOT6 [2]; Nguyễn Văn Thị nghiên cứu

Ngày nhận bài: 01/03/2018, ngày chuyển phản biện: 05/03/2018, ngày chấp nhận phản biện: 20/03/2018, ngày chấp nhận đăng: 22/3/2018 
phân loại trạng thái rừng bằng phương pháp phân loại hướng đối tượng trên tư liệu ảnh vệ tinh SPOT5 [5]... Tư liệu viễn thám chủ yếu trong các nghiên cứu là dữ liệu ảnh vệ tinh có độ phân giải cao, độ phân giải siêu cao, chưa có nhiều nghiên cứu thử nghiệm trên ảnh UAV. Do đó, nội dung bài báo sẽ là kết quả bước đầu trong nghiên cứu phân loại tư liệu ảnh UAV phục vụ thành lập các loại bản đồ chuyên đề tỷ lệ lớn, tự động phát hiện, giải đoán các mục tiêu quân sự.

\section{Tư liệu và khu vực nghiên cứu}

\subsection{Khu vực nghiên cứu}

Khu vực nghiên cứu là cảng Hàng không quốc tế (HKQT) Tân Sơn Nhất và được thể hiện trong Hình 1. Cảng HKQT Tây Sơn Nhất nằm ở vị trí tọa độ $10^{\circ} 49^{\prime} 13.63^{\prime \prime} \mathrm{N}$ và 10660'39.23"E (trong hệ tọa độ WGS-84) với độ cao là $10 \mathrm{~m}$ so với mực nước biển trung bình. Đây là khu vực có nhiều đối tượng địa vật như đường băng, sân đỗ, nhà ga, máy bay. Các đối tượng địa vật có sự khác biệt về phổ do có độ cao khác nhau, màu sắc khác nhau và góc chụp khác nhau. Vì vậy, khu vực nghiên cứu sẽ giúp đánh giá được những ưu và nhược điểm khi áp dụng phương pháp phân loại hướng đối tượng trên tư liệu ảnh viễn thám độ phân giải siêu cao, đặc biệt là ảnh UAV. (Xem hình 1)

\subsection{Tư liệu nghiên cứu}

Tư liệu nghiên cứu là ảnh máy bay không người lái UX5 với hệ thống máy ảnh kỹ thuật số độ phân giải không gian là $40 \mathrm{~cm}$. Thời gian chụp là 1:44:38 PM ngày 29/12/2016. Dữ liệu ảnh gồm có 3 kênh phổ trong dải sóng nhìn thấy là kênh đỏ (Red); kênh xanh lục (Green) và kênh xanh chàm (Blue) (Hình 1c).

\section{Phương pháp nghiên cứu và kết quả thử nghiệm}

\subsection{Phương pháp nghiên cứu}

Phân tích các đối tượng trên Hình 1c, tác giả lựa chọn các đối tượng chính cần phân loại trên ảnh gồm đường băng, sân đỗ, nhà gần sân bay, máy bay (gồm máy bay đang cất cánh và máy bay đỗ tại đường băng). Trong nội dung bài báo, tác giả sử dụng phần mềm eCognition 9.0 để thử nghiệm. Quy trình phân loại hướng đối tượng trên ảnh UAV được thể hiện trong Hình 2.

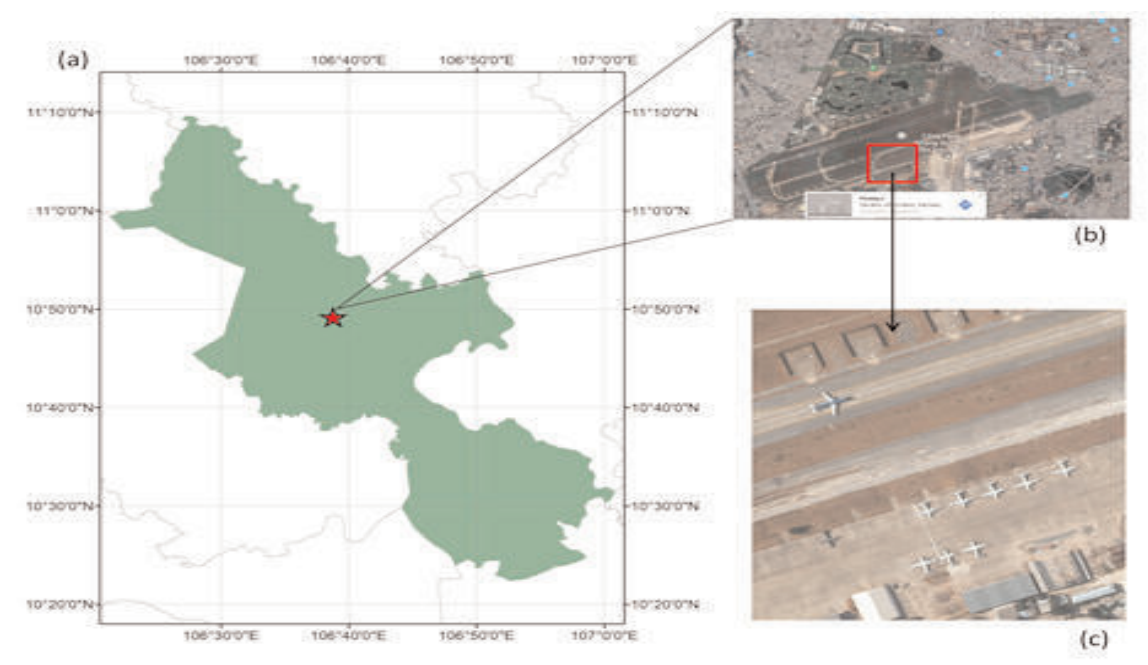

Hình 1: Khu vực nghiên cứu

(a) Ranh giới hành chính của Thành phố Hồ Chí Minh. (b) Toàn cảnh của Cảng hàng không quốc tế Tân Sơn Nhất. (c) Vị trí khu vực nghiên cứu 
Bước đầu tiên trong phương pháp phân loại hướng đối tượng là tự động phân vùng các đối tượng trên ảnh. Kết quả phân vùng đối tượng sẽ quyết định độ chính xác các bước tiếp theo trong quy trình phân loại. Trong nội dung nghiên cứu của bài báo, tác giả sử dụng phương pháp phân vùng ảnh đa độ phân giải (multiresolution segmentation). Phương pháp phân vùng ảnh cần xác định các thông số (Scale parameter, Shape, Compactness) phù hợp đối với từng tư liệu ảnh. Trên Hình 3 và Bảng 1 thể hiện các thông số và kết quả được thử nghiệm trên ảnh UAV. Kết quả thử nghiệm cho thấy trường hợp 4 phân vùng tốt các đối tượng trên ảnh, đặc biệt là đối tượng máy bay, nhà.

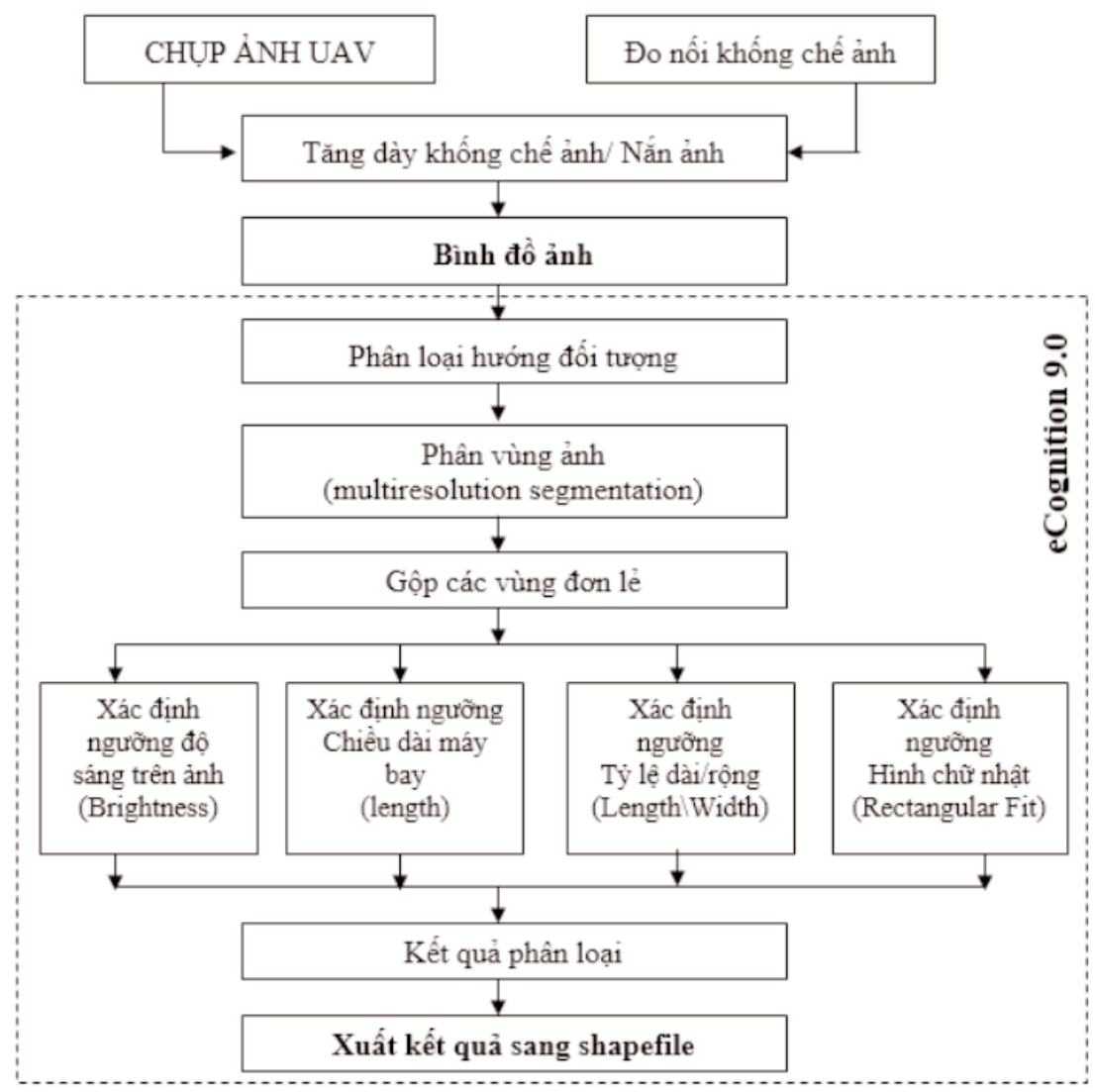

Hình 2: Quy trình phân loại hướng đối tượng trên ảnh UAV

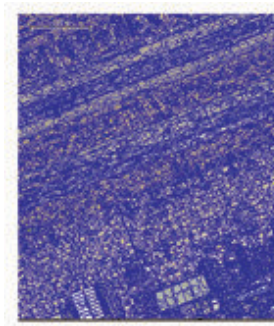

Trường hợ 1

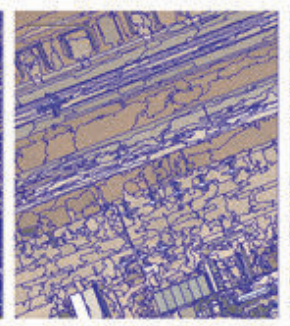

Trường hợp 2

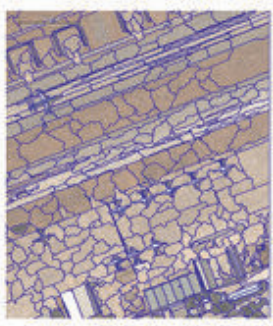

Trường hợp 3

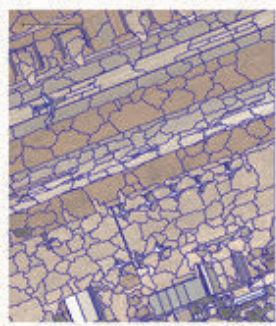

Trường hợp 4

Hình 3: Kết quả thử nghiệm các thông số phân vùng trên ảnh UAV 
Bảng 1: Các thông số thử nghiệm phân vùng ảnh

\begin{tabular}{|c|c|c|c|}
\hline PP phân vùng 1 & \multicolumn{3}{|c|}{ Các thông số phân vùng ảnh } \\
\cline { 2 - 4 } phân giải & Scale parameter & Shape & Compactness \\
\hline Trường hợp 1 & 10 & 0.1 & 0.5 \\
\hline Trường hợp 2 & 50 & 0.1 & 0.5 \\
\hline Trường hợp 3 & 50 & 0.5 & 0.5 \\
\hline Trường hợp 4 & 50 & 0.5 & 0.7 \\
\hline
\end{tabular}

Bảng 2: Tỷ lệ chiều dài thân và sải cánh của các loại máy bay

\begin{tabular}{|c|c|c|c|c|c|}
\hline \multirow{2}{*}{ TT } & \multirow{2}{*}{$\begin{array}{l}\text { Loại máy } \\
\text { bay }\end{array}$} & \multicolumn{3}{|c|}{ Chỉ số của máy bay thụ̣c tế } & \multirow{2}{*}{$\begin{array}{c}\text { Tỷ lệ xác định trên } \\
\text { ảnh }\end{array}$} \\
\hline & & Chiều dài thân & Chiều dài sải cánh & Tỷ lệ thân/cánh & \\
\hline 1 & Boeing 787 & 63.73 & 60.93 & 1.0460 & \multirow{6}{*}{$\begin{array}{c}\text { Giá trị nhỏ nhất }= \\
1.172 \\
\text { Giá trị lớn nhất }= \\
1.512\end{array}$} \\
\hline 2 & AirBus A350 & 66.89 & 64.75 & 1.0331 & \\
\hline 3 & AirBus A330 & 63.6 & 60.3 & 1.0547 & \\
\hline 4 & AirBus A321 & 44.51 & 34.1 & 1.3053 & \\
\hline 5 & AirBus A320 & 37.573 & 34.156 & 1.1000 & \\
\hline 6 & Boeing 787-8 & 56.7 & 60.1 & 0.9434 & \\
\hline
\end{tabular}

Bước tiếp theo của quy trình phân loại là tách các đối tượng riêng biệt trên ảnh. Đối với các đối tượng dạng mảng như đường băng, sân đỗ, tác giả sử dụng chỉ số ngưỡng độ sáng (Brightness) trên ảnh để phân loại. Đối tượng nhà có hình mái chữ nhật sẽ sử dụng chỉ số ngưỡng Rectangular fit. (Xem bảng 2)

Các đối tượng máy bay trên sân đỗ sẽ sử dụng chỉ số tỷ lệ dàilrộng (LengthIWidth). Chỉ số tỷ lệ dàilrộng tương đối phù hợp để phân loại các đối tượng máy bay do kích thước các loại máy bay được công bố thông qua hai chỉ số là chiều dài thân và chiều dài sải cánh (tham khảo bảng 2). Trên ảnh có một đối tượng máy bay đang trên đường băng có dạng màu sắc và kích thước khác biệt nên dùng thêm các chỉ số chiều dài (length) để phân loại. Kết quả phân loại hướng đối tượng trên ảnh UAV được thể hiện trong Hình 4.

\section{Kết luận}

Phần mềm eCognition 9.0 cho phép phân loại hướng đối tượng trên các tư liệu viễn thám có độ phân giải cao, độ phân giải siêu cao như ảnh UAV. Kết quả phân loại hướng đối tượng trên ảnh UAV cần lựa chọn tham số phân vùng đối tượng với kích thước Scale parameter, Shape, Compactness lần lượt là $50 ; 0.5 ; 0.7$. Các đối tượng trên ảnh được phân loại dựa theo đặc điểm hình học hoặc độ sáng trên ảnh... Tuy nhiên, đối với những đối tượng có độ cao sẽ tạo bóng vật thể, gây nhầm lẫn với các đối tượng thực. Các thông tin được phân tích trên ảnh xuất sang shapefile sẽ thuận lợi cho việc biên tập tạo các sản phẩm bản đồ địa hình, bản đồ chuyên đề, cập nhật vào cơ sở dữ liệu địa vật phục vụ cho việc tự động phát hiện và nhận dạng các mục tiêu quân sự từ tư liệu viễn thám độ phân giải siêu cao. 0

\section{Tài liệu tham khảo}

[1]. T. Blaschke (2010). Review article object based image analysis for remote sensing. ISPRS Journal of Photogrammetry and Remote Sensing 65(2010) 2-16.

[2]. Phạm Văn Duẩn, Vũ Thị Thìn, Nguyễn Quốc Huy (2016). U'ớc tính giá trị các thông số khoanh vi ảnh hướng đối tượng phù hợp trên phần mềm eCognition: 


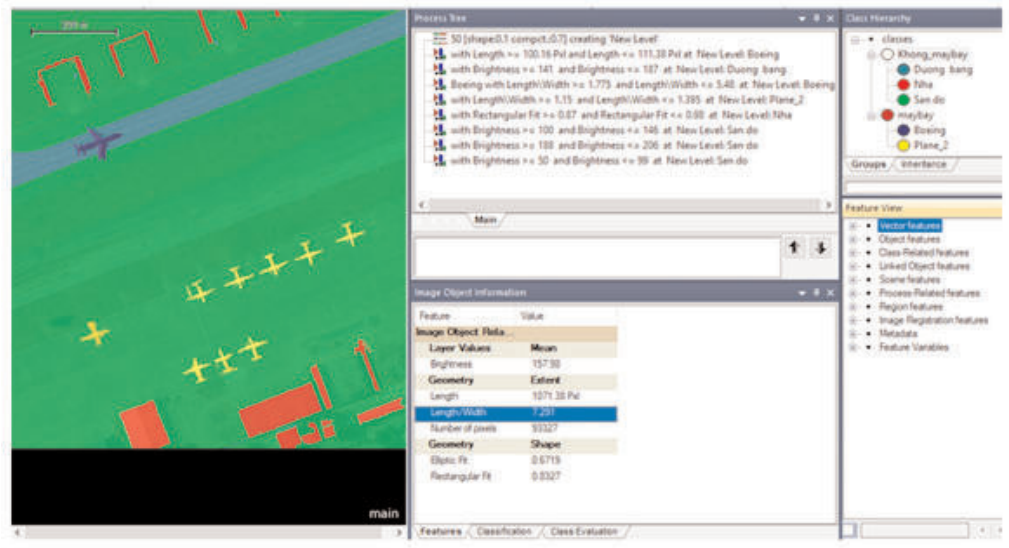

Hình 4: Kết quả phân loại trên phần mềm eCognition 9.0

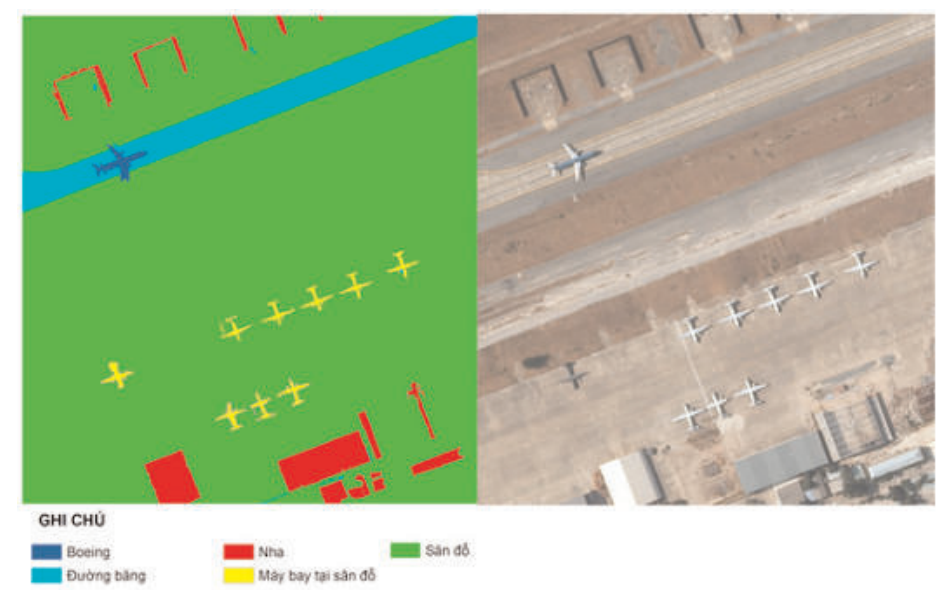

Hình 5: Kết quả phân loại so với ảnh dữ liệu gốc
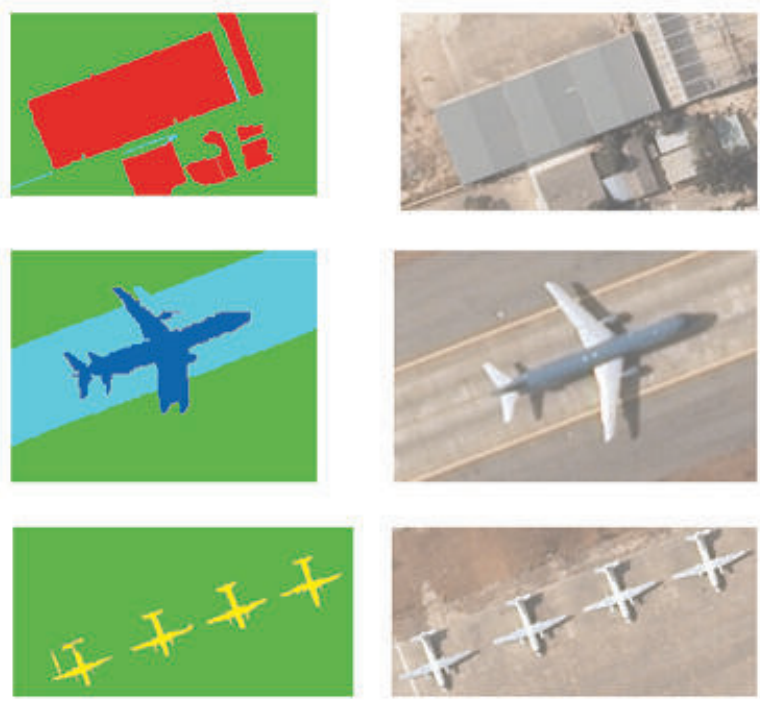

Hình 6: So sánh kết quả phân loại các đối tượng trên ảnh UAV 
Thử nghiệm với ảnh vệ tinh SPOT 6. Tạp chí quản lý Tài nguyên rừng \& Môi trường số 6 - 2016.

[3]. Miao Li, Shuying Zang, Bing Zhang, Shanshan Li \& Changshan Wu (2014). A review of remote sensing image classification techniques: the Role of Spatio-contextual information. European Journal of Remote Sensing 2014, 47: 389-411.

[4]. Xiaoxiao Li, Guofan Shao (2014). Object-Based land-cover mapping with high resolution aerial photography at a county scale in Midwestern USA. Remote sensing. 2014, 6. 11372-11390; doi:10.3390/rs61111372.

[5]. Nguyễn Văn Thị, Trần Quang Bảo (2014). Ứng dụng kỹ thuật phân loại ảnh hướng đối tượng nhằm phân loại trạng thái rừng theo thông tư số 34 . Tạp chí Khoa học lâm nghiệp số 2/2014 (3343-3353).

[6]. Trịnh Thị Hoài Thu (2012). So sánh phương pháp phân loại dựa vào điểm ảnh và phân loại định hướng đối tượng chiết xuất thông tin lớp phủ bề mặt từ ảnh độ phân giải cao. Tạp chí Khoa học kỹ thuật Mỏ - Địa chất, số 39, 7/2012, (Chuyên đề Trắc địa mỏ), tr.59-64.

[7]. Yan Xu, Fuzhou Duan (2013). Color space transformation and object oriented based information extraction of aerial images. 21st International Conference on Geoinformatics, 2013.

[8]. Wenxia Wei, Xiuwan Chen, Ainai Ma (2005). Object-oriented Information Extraction and Application in High-resolution Remote Sensing image. IEEE 2005. O

\section{Summary}

\section{Object-oriented classification and application in UAV images}

\section{Le Vu Hong Hai, Military of Technical Academy}

Do Thi Hoai, Institute of Geodesy and Cartography

\section{Vu Ky Long, Hanoi Environment and Natural Resources Department}

Unmanned aerial vehicles (UAVs) collect information at low altitude, so multi-color images had obvious characteristics and fitted for visual interpretation. UAV data were lacking of spectral information but rich shape and texture information of the objects. However, the reality was that there was less study on extraction of ground surface information from UAV images due to the low accuracy by pixel-based classification. In the article, the author presented application of object-oriented classification using eCognition software 9.0 in UAV image data which spatial resolution was $40 \mathrm{~cm}$. Object-oriented classification allows to extract information of the objects in very high resolution images based on shape characteristic features with high accuracy. $O$ 